1989, 38, 1

\title{
СХЕМА ДЕКОМПОНИРОВАННОГО УПРАВЛЕНИЯ НЕЛИНЕЙНОЙ ДИСКРЕТНО-НЕПРЕРЫВНОЙ СИСТЕМОЙ
}

\author{
I. RANDVEE. KAHENIVOOLINE SKEEM AJAS DISKREETSETE MITTELINEAARSETE SUSTEE- \\ MIDE JUHTIMISEKS \\ I. RANDVEE. TWO-LEVEL CONTROL LAW FOR NASH-OPTIMAL NONLINEAR DISCRETE- \\ TIME SYSTEMS
}

\author{
(Представил Ю. Яаксоо)
}

Предлагается алгоритм формирования сигнала управления для линейной относительно управлений и нелинейной относительно вектора состояния дискретно-непрерывной системы, реализующий в ней равновесное состояние по Нэшу. Алгоритм основан на выдвинутом в работе ['] приеме, который позволяет обойти понятие сопряженной переменной при использовании необходимых условий оптимальности.

\section{1. Постановка задачи}

Дана дискретно-непрерывная система в виде однозначной линейной относительно управлений вектор-функции:

$$
x_{k+1}=f\left(x_{k}\right)+\sum_{i=1}^{m} B_{i} u_{i k}, \quad k=0,1, \ldots, N-1,
$$

где $x_{k} \in R^{n}, u_{i k} \in R^{r_{t}}, r_{i} \leqslant n, B_{i}$ - известные числовые матрицы.

Задача состоит в нахождении последовательностей управлений $u_{i k}$, $i=1,2, \ldots, m ; k=0,1, \ldots, N-1$, которые при фиксированном начальном состоянии $x_{0}$ удовлетворяют уравнению движения (1) и минимизируют критерий

$$
J_{i}=\frac{1}{2} \sum_{k=0}^{N-1}\left(x_{k}^{\prime} Q_{i} x_{k}+u_{i k}^{\prime} R_{i} u_{i k}\right)+\frac{1}{2} x_{N}^{\prime} Q_{i} x_{N},
$$

где $Q_{i} \geqslant 0, R_{i}>0$ известные симметричные матрицы. На управления $u_{i k}$ никаких ограничений не накладывается.

Учитывая, что каждый локальный критерий (2) зависит (через общий вектор состояния) от всех реализованных управлений, можем записать:

$$
J_{i}\left(U_{1}^{*}, \ldots, U_{m}^{*}\right) \leqslant J_{i}\left(U_{1}^{*}, \ldots, U_{i-1}^{*}, U_{i}, U_{i+1}^{*}, \ldots, U_{m}^{*}\right)
$$

для всех $i$, где $U_{i}=\left(u_{i 0}^{\prime}, \ldots, u_{i, N-1}^{\prime}\right)^{\prime}, U_{i}^{*} \quad$ - оптимальное управление, т. е. в результате минимизации локальных функций цели (2) в системе (1) устанавливается равновесное состояние по Нэшу [2].

\section{2. Метод решения}

Для решения задачи (1), (2) целесообразно использовать новый метод декомпозиции, примененный для задач статики большой размерности $\left[{ }^{1}\right]$. Сущность метода (в терминах и обозначениях данной за- 
дачи) состоит в следующем. Из критерия $J_{i}(2)$ выделяется определенная часть

$$
J_{i}=J_{i k}+\bar{J}_{i k}, \quad k=0,1, \ldots, N-1 .
$$

Необходимое условие оптимальности $J_{i}$ по $u_{i k}$ записывается в следующем виде:

$$
d J_{i k} / d u_{i k}+g_{i k}=0,
$$

где $g_{i k}$ - векторный параметр, зависящий от $u_{i k}$ неявно, а символ $d J_{i k} / d u_{i k}$ означает частное производное $J_{i k}$ по переменной $u_{i k}$.

Предполагается, что матрицы частных производных $d x_{k+1} / d x_{k}$, $d x_{k+1} / d u_{i k}$ существуют, причем первая из них должна быть неособенной, а значение второй известно. Как видно из уравнения (1) в нашем случае $d x_{k+1} / d u_{i k}=B_{i}$. Кроме того, предполагается, что движение системы, определяемое уравнениями (1) и (3) при $g_{i k}=0$ однозначное и устойчивое.

Далее, для решения уравнения (3) строится простой итерационный процесс: на устойчивой траектории, определенной уравнениями (1) и (3), вычисляется новое значение параметра ( $g_{i k}=0-$ начальное значение)

$$
g_{i k}=\left(d x_{k+1} / d u_{i k}\right)^{\prime} d \bar{J}_{i k} / d x_{k+1},
$$

которое подставляется в (3) для получения уточненного управления и уточненного движения. Сходимость итерационного процесса гарантируется выпуклостью критерия (2) и выполнением вышеуказанных предположений.

\section{3. Алгоритм решения}

Декомпонируем критерий $J_{i}$, заданный выражением (2), так, чтобы

$$
\begin{aligned}
J_{i k}=\frac{1}{2}\left(x_{k+1}^{\prime} Q_{i} x_{k+1}+u_{i k}^{\prime} R_{i} u_{i k}\right), \\
k=0,1, \ldots, N-1 .
\end{aligned}
$$

Уравнение (3), согласно декомпозиции (4), приводит к закону управления в частично-замкнутом виде:

$$
u_{i k}=-D_{i} B_{i}^{\prime} Q_{i} f\left(x_{k}\right)+v_{i k},
$$

где $D_{i}=\left(B_{i}^{\prime} Q_{i} B_{i}+R_{i}\right)^{-1}$,

$$
v_{i k}=-D_{i}\left[B_{i}^{\prime} Q_{i} \sum_{j=1}^{m} B_{j} u_{j k}+g_{i k}\right], \quad i=1,2, \ldots, m ; i \neq j .
$$

В частности, закон управления для линейного объекта получим, если в (5) $f\left(x_{k}\right)$ заменим на $A x_{k}$, где $A-n \times n$ известная матрица.

Значение параметра $v_{i k}$ уточняется вышеописанными итерациями. При этом можно использовать одну из следующих схем:

a) Вычисляется траектория движения системы (1) при управлении (5) и при фиксированном значении параметра $v_{i k}$ (начальное значение $v_{i k}=0$ )

$$
x_{k+1}=\bar{f}\left(x_{k}, v_{1 k}, \ldots, v_{m k}\right) .
$$

На этой траектории вычисляется, начиная с $k=N$, значение $g_{i k}$ :

$$
g_{i k}=B_{i}^{\prime} G_{i k},
$$

где

$G_{i, k-1}=\left(d \bar{f} / d x_{k}\right)^{\prime} Q_{i} x_{k+1}+\left(d h_{i k} / d x_{k}\right)^{\prime} R_{i} u_{i k}+\left(d \bar{f} / d x_{k}\right)^{\prime} G_{i k}, \quad G_{i N}=0$, 
при значениях $x_{k}$, заданных уравнением (7), и при $u_{i k}$, заданном уравнением (5), $h_{i k}=-D_{i} B_{i}^{\prime} Q_{i} f\left(x_{k}\right)$. Для линейного объекта

$$
G_{i, k-1}=F^{\prime} Q_{i} x_{k+1}+A_{i}^{\prime} R_{i} u_{i k}+F^{\prime} G_{i k}, \quad G_{i, N}=0,
$$

где

$$
\begin{aligned}
F & =\left(A+\sum_{i=1}^{m} B_{i} A_{i}\right), \\
A_{i} & =-D_{i} B_{i}^{\prime} Q_{i} A, \quad i=1,2, \ldots, m .
\end{aligned}
$$

Новое уточненное значение $v_{i k}$ задается уравнением (6), в котором используются найденные $g_{i k}$ и управления $u_{j k}$ предыдущего шага итерации.

б) Вторая схема уточнения $v_{i k}$ отличается от первой тем, что фиксируется только параметр $g_{i k}$. Система (5)-(6) решается относительно $u_{i k}$ на каждом шаге уточнения траектории движения одним из известных методов, например, с помощью метода покоординатного спуска. По принятым ранее предположениям решение системы уравнений (5)-(6) существует, т. е. итерационный процесс по $u_{i_{k}}$ можно считать сходящимся в обеих предлагаемых схемах.

Для улучшения сходимости итерационного процесса как по $u_{i k}$, так и по $g_{i k}$ целесообразно вместо простой подстановки использовать релаксацию $\left[{ }^{1}\right]$

$$
g_{i k}^{s}=W g_{i k}+(I-W) g_{i k}^{s-1},
$$

где $g_{i k}$ - вычисленное по (8) или (9) значение вектора параметров, $W$ - релакционная матрица, $s$ - номер итерации.

Нужно отметить, что выражение (5) представляет собой составной регулятор. Часть сигнала управления, которая зависит от текущего состояния, основывается на минимизации критерия (4) и представляет, как известно $\left(\left[{ }^{3}\right]\right.$, с. 123$)$, регулятор состояния с одношаговым установлением, т. е. сигнал управления рассчитан для ликвидации отклонения от желаемого состояния за один шаг. Сигнал управления в подобных регуляторах, хотя и принимает нежелательно большие значения, остается ограниченным. Несмотря на это подобные регуляторы весьма широко используются $\left[{ }^{3,4}\right]$. Остальная часть в законе (5) представляет собой разомкнутое (программное) управление, которое учитывает влияние всего интервала оптимизации (и остальных управлений). Эта часть уточняется итеративно.

Так как замкнутая система предполагается устойчивой, то сигнал управления может быть реализован на объекте на любом шаге итерационного процесса. Последнее обстоятельство облегчает применение данного алгоритма в управляющих системах реального времени.

\section{4. Примеры для иллюстрации}

В таблице приведены значения критерия $J_{i}$ вида (2), полученные в ходе уточнения сигнала управления по первой схеме предложенного алгоритма для линейной системы (I), линейных относительно управлений систем (II) и (III)

$$
\begin{aligned}
& x_{k+1}=a x_{k}+b_{1} u_{1 k}+b_{2} u_{2 k}, \quad x_{0}, \\
& x_{k+1}=a\left(1+x_{k}^{2}\right)^{-1} x_{k}+b_{1} u_{1 k}+b_{2} u_{2 k}, \quad x_{0}, \\
& x_{k+1}=a x_{k}^{3}+b_{1} u_{1 k}+b_{2} u_{2 k}, \quad x_{0} .
\end{aligned}
$$




\begin{tabular}{c|c|c|c|c|c|c|c|c|c|c}
\hline \multirow{s}{*}{$\boldsymbol{s}$} & 1 & 2 & 3 & 4 & 5 & 6 & $J_{1} / J_{90}$ & $J_{0}$ & $a$ & $W_{\boldsymbol{B}}$ \\
\hline \multirow{2}{*}{ I } & 1,6 & 1,4 & 1,1 & 1,1 & 1,1 & 1,1 & 1,45 & 1,0 & 1,2 & 0,4 \\
& 2,2 & 1,5 & 1,3 & 1,4 & 1,3 & 1,3 & 1,69 & 1,2 & 1,3 & 0,4 \\
II & 0,5 & 0,4 & 0,4 & 0,4 & 0,4 & 0,4 & 1,25 & & 1,3 & 0,5 \\
& 0,9 & 0,8 & 0,7 & 0,7 & 0,7 & 0,7 & 1,28 & & 1,5 & 0,3 \\
III & 1,4 & 1,6 & 1,2 & 1,3 & 1,3 & 1,3 & 1,08 & & 1,4 & 1,5 \\
& 2,9 & 1,5 & 1,8 & 1,8 & 1,7 & 1,7 & 1,70 & & 1,6 & 0,3
\end{tabular}

$B_{1}=B_{2}=0,5 ; \quad g_{1}=g_{2}=r_{1}=r_{2}=1,0$;

$X_{0}=1,0 ; W_{u}=0,9 ; N=20$.

$J_{0}$ - означает минимальное значение критерия для задачи (1), (2), полученное аналитически $\left[{ }^{5}\right], J_{1}-$ значение критерия при $g_{i k}=0$.

Данные таблицы указывают на улучшение сходимости процесса уточнения сигнала управления и на некоторое уменьшение значимости ее разомкнутой части (характеризуемое отношением значений $J_{i}$ при $s=1$ и $s=90)$ при увеличении степени устойчивости замкнутой обратной связью системы. Нужно отметить, что сходимость в значительной степени зависит от значений релаксационных параметров. При правильном их выборе итерационный процесс можно считать законченным за несколько (3-6) шагов.

Предлагаемая схема может быть полезной, подобно методу статических взаимосвязей $\left[{ }^{6}\right]$, в системах управления взаимосвязанными объектами (котельной установкой, установкой производства бумаги и т. п.), которыми управляют локальные несвязанные регуляторы.

\section{ЛИТЕРА Т Р А}

1. Drouin, M., Abou-Kandil, H., Mariton, M., Duc, G. // APII, 1985, 19, № 3, 205-226.

2. Жуковский В. Н., Стоянов Н. В. // Годишн. Висш. учебни завед. Прилож. мат., 1984/1985. 20. № 4. 9-23

3. Goodwin, G. C., Sin, K. S. Adaptive Filtering Prediction and Control. PrenticeHall, Englewood Cliffs (N. J.), 1984.

4. Нзерман Р. Н. Цифровые системы управления. М, 1984.

5. Рандвеэ И. // Изв. АН ЭССР. Физ. Мат., 1986, 35, № 1, 107-109.

6. Chang. T. N., Davison, E. J. Proc. 25th IEEE Conf. Decis. and Contr. Athens, Dec. $10-12,1986$. New York, 1986, 2, 881-887.

Институт кибернетики

Академии наук Эстонской ССР
Поступила в редакцию $12 /$ IV 1988 